BULL. AUSTRAL. MATH. SOC.

$49 A 22,49 B 22,93 C 20$

VOL. 27 (1983), 139-148.

\title{
ON AN OPTIMAL CONTROL PROBLEM INVOLVING \\ SECOND ORDER HYPERBOLIC SYSTEMS \\ WITH BOUNDARY CONTROLS
}

\author{
K.G. ChOo, K.L. TEO AND Z.S. Wu
}

\begin{abstract}
In this paper, we consider an optimal control problem involving second-order hyperbolic systems with boundary controls. Necessary and sufficient conditions are derived and a result on the existence of optimal controls is obtained. Also, a computational algorithm which generated minimizing sequences of controls is devised and the convergence properties of the algorithm are investigated.
\end{abstract}

\section{Introduction}

In Section 4.2 of [1], a class of problems of optimal control of systems governed by a second-order hyperbolic partial differential equation with Dirichlet boundary conditions had been studied. This class of optimal control problems arises naturally in the study of optimal generation and transmission of power over large networks, and the results are originally due to Ahmed [2].

In this paper, we consider a similar class of optimal control problems. More precisely, our system is the same as that considered in [1] and [2]; the cost functional is, however, slightly more general. Following the steps of [3] and [4], our aims are:

Received 5 October 1982. This work, which is partially supported by the Australian Research Grants Committee, was done during the period when K.G. Choo and Z.S. Wu were Honorary Visiting Fellows in the School of Mathematics at the University of New South Wales. 
(i) to derive a necessary and sufficient condition for optimality ;

(ii) to prove a result on the existence of optimal controls;

(iii) to devise a computational algorithm;

(iv) to investigate certain convergence properties of the algorithm.

The results on the questions (i) and (ii) can be considered as slight extensions of those counterparts presented in Section 4.2 of [1].

The algorithm of this paper is very close to that of Section 4.2 of [1] and that of [2]. However, the differences are rather crucial, because we can prove certain important convergence properties of our algorithm, which are otherwise not possible to obtain using either the approach of [2] or that of Section 4.2 of [1].

\section{The optimal control problem}

Let $Z_{0}$ and $T$ be positive constants, $\Omega \equiv\left(0, Z_{0}\right), I \equiv(0, T)$ be the time interval of interest, and $Q \equiv \Omega \times I$. Let $\partial \Omega \equiv\left\{0, \tau_{0}\right\}$ denote the end points of $\Omega$ and let $\Sigma \equiv \partial \Omega \times I$.

Consider the distributed system governed by the following hyperbolic equation

$$
\begin{array}{rlrl}
\phi_{t t}(x, t)+a \phi_{t}(x, t)+b \phi(x, t)-c \phi_{x x}(x, t) & =\alpha f_{x}(x, t), & (x, t) \in Q, \\
\phi(x, 0) & =\phi_{0}(x), & x \in \Omega, \\
\phi_{t}(x, 0) & =\phi_{1}(x), & & x \in \Omega, \\
\phi(1) & & t \in I, \\
\phi\left(z_{0}, t\right) & =u_{1}(t), & \\
& & t \in \in I,
\end{array}
$$

where $u \equiv\left(u_{1}, u_{2}\right)$ is a 2-control vector, and $a, b, c, \alpha$ are constants.

Let $V$ be a fixed compact and convex subset of $R^{2}$. Any measurable function $u \equiv\left(u_{1}, u_{2}\right)$ from $I$ into $V$ is called an admissible control. 
We denote by $U$ the class of all such admissible controls.

To proceed further, we need to introduce some more notation. First, we let

$$
F^{*}(\psi) \equiv \psi_{t t}-a \psi_{t}+b \psi-c \psi_{x x}
$$

Then, let $X$ be the subset of $W_{2}^{1,1}(Q)$ given by

(2) $\quad X \equiv\left\{\psi \in W_{2}^{1,1}(Q) \mid \psi(x, T)=\psi_{t}(x, T)=0\right.$ for $x \in \Omega, \psi(x, t)=0$ for $(x, t) \in \Sigma$ and $\left.F^{*}(\psi) \in L_{2}(Q)\right\}$.

Note that $X$ equipped with the norm topology \|\|$\psi\|\|_{X}=\left\|F^{*}(\psi)\right\|_{2, Q}$ is a Hilbert space.

For the system (1), the following conditions are assumed throughout the paper:

(Al) $a>0, b>0, c>0$ and $\alpha$ any real number;

(A2) $\phi_{0} \in W^{l}(\Omega), \phi_{1} \in L_{2}(\Omega), f \in L_{2}(Q)$;

(A3) $\int_{I}\left\{u_{1}(t) \Psi_{x}(0, t)-u_{2}(t) \psi_{x}\left(Z_{0}, t\right)\right\} d t<\infty$, for all $\psi \in X$ and for all $u \in U$ :

REMARK 2.1. It follows from Theorem 4.2.1 of [1], that the system (1) admits a unique generalized solution $\phi(u) \in L_{2}(Q)$, for each $u \in U$. (For the definition of generalized solution, we refer to Definition 4.2.2 of [1].)

REMARK 2.2. By Lemma 4.2.2 of [1], we see that the generalized solution $\phi(u)$ of the system (1) corresponding to $u \in U$ has a unique weak linear Gateaux differential at every point $u \in U$ in any direction $w=v-u$ with $v \in U$. This differential is the generalized solution $\hat{\phi}(w) \in L_{2}(Q)$ of the following system:

$$
\begin{aligned}
\hat{\phi}_{t t}(x, t)+a \hat{\phi}_{t}(x, t)+b \hat{\phi}(x, t)-c \hat{\phi}_{x x}(x, t) & =0, \quad(x, t) \in Q, \\
\hat{\phi}(x, 0)=\hat{\phi}_{t}(x, 0) & =0, x \in \Omega, \\
\hat{\phi}(x, t) & =\omega, \quad(x, t) \in \Sigma .
\end{aligned}
$$


Moreover, the map $w \rightarrow \hat{\phi}(w)$ is linear.

Now let $h(x, t, y)$ and $g(t, u)$ be real-valued continuous functions defined on $Q \times R$ and $I \times R^{2}$ respectively. We assume that these functions are twice differentiable and convex with respect to the variables $y$ and $u$ respectively. We denote by $\nabla h$ and $\nabla^{2} h$, respectively, the partial derivatives of the first and second order of $h$ with respect to $y$. Let $\nabla g$ and $\nabla^{2} g$ be the gradient and Hessian of $g$ with respect to $u$. In addition we assume that $\nabla h(\cdot, \cdot, \phi(u)(\cdot, \cdot)) \in L_{2}(Q)$, for any $u \in U$, where $\phi(u)$ is the generalized solution of the system ( 1 ) corresponding to the control $u \in U$.

The cost functional is defined by

$$
J(u)=\iint_{Q} h(x, t, \phi(u)(x, t)) d x d t+\int_{I} g(t, u(t)) d t .
$$

The optimal control problem is then to minimize $J(u)$ subjected to the system (1). Such control problem will be referred to as Problem (P).

We now consider the following adjoint system of Problem ( $P$ ), which is a homogeneous boundary value problem:

$$
\begin{aligned}
& z_{t t}(x, t)-a z_{t}(x, t)+b z(x, t)-c z_{x x}(x, t)=\nabla h(x, t, \phi(u)(x, t)), \\
& \text { (or just } \left.F^{*}(z)(x, t)=\nabla h(x, t, \phi(u)(x, t))\right), \quad(x, t) \in Q, \\
& z(x, T)=z_{t}(x, T)=0, x \in \Omega, \\
& z(x, t)=0, \\
& \begin{array}{ll}
(5) & (x, t) \in \Sigma,
\end{array}
\end{aligned}
$$

where $\phi(u)$ is the generalized solution of the system (1) corresponding to the control $u \in U$.

REMARK 2.3. Since. $\nabla h(\cdot, \cdot, \phi(u)(\cdot, \cdot)) \in L_{2}(Q)$ for each $u \in u$, it follows from Lemma 4.2 .1 of [1] that the adjoint system (5) has a unique generalized solution $z(u) \in W_{2}^{\perp, l}(Q)$ for each $u \in U$. In fact, from the definition of $X$ and (5), we see that such $z(u)$ is in $X$.

LEMMA 2.1. Consider the problem $(\mathrm{P})$. Then $u^{*} \in U$ is an optimal control if and only if $u^{*}$ minimizes the functional 
(6) $\quad J_{u^{*}}^{\prime}(u) \equiv \iint_{Q} \hat{\phi}(u)(x, t) \nabla h\left(x, t, \phi\left(u^{*}\right)(x, t)\right) d x d t$

$$
+\int_{I} u(t) \nabla g\left(t, u^{*}(t)\right) d t
$$

where $\hat{\phi}(u)$ is the generalized solution of the system (3) corresponding to the control $u \in U$.

The proof of this lemma is standard.

Using Lemma 1.1 and modifying the proof of Lemma 4.2.2 of [1] appropriately, we obtain:

THEOREM 2.1. Consider Problem (P). Then $u^{*} \equiv\left(u_{1}^{*}, u_{2}^{*}\right) \in u$ is an optimal control if and only if

$$
\text { (7) } \begin{aligned}
& \int_{I}\left\{c\left\{\left(u_{1}(t)-u_{1}^{*}(t)\right)\left(z\left(u^{*}\right)\right)_{x}(0, t)-\left(u_{2}(t)-u_{2}^{*}(t)\right)\left(z\left(u^{*}\right)\right)_{x}\left(z_{0}, t\right)\right\}\right. \\
&\left.+\left(u(t)-u^{*}(t)\right) \nabla_{g}\left(t, u^{*}(t)\right)\right\} d t \geq 0,
\end{aligned}
$$

for azz $u \equiv\left(u_{1}, u_{2}\right) \in u$.

A pointwise version of the necessary and sufficient condition (7) is given below as a corollary.

COROLLARY 2.1. Consider Problem $(P)$. Let $u^{*} \equiv\left(u_{1}^{*}, u_{2}^{*}\right) \in U$. Then the necessary and sufficient condition for $u^{*}$ to be an optimal control is that the inequality

(8) $\left\{c\left\{\left(v_{1}-u_{1}^{*}(t)\right)\left(z\left(u^{*}\right)\right)_{x}(0, t)-\left(v_{2}-u_{2}^{*}(t)\right)\left(z\left(u^{*}\right)\right)_{x}\left(z_{0}, t\right)\right\}\right.$

$$
\left.+\left(v-u^{*}(t)\right) g\left(t, u^{*}(t)\right)\right\} \geq 0
$$

holds for almost all $t \in I$ and for all $v \equiv\left(v_{1}, v_{2}\right) \in V$.

It is not difficult to observe that the cost functional $J(u)$ given by (4) satisfies

$$
\begin{array}{r}
J(u)-J(w) \geq \int_{I}\left\{c\left\{\left(u_{1}(t)-w_{1}(t)\right)(z(w))_{x}(0, t)-\left(u_{2}(t)-w_{2}(t)\right)(z(w))_{x}\left(l_{0}, t\right)\right\}\right. \\
+(u(t)-w(t)) \nabla g(t, w(t))\} d t,
\end{array}
$$

for all $u \equiv\left(u_{1}, u_{2}\right), w \equiv\left(w_{1}, w_{2}\right) \in u$. Thus, in particular, we have 


$$
\begin{aligned}
J(u) \geq & J(0) \\
& \left.+\int_{I}\left\{c\left\{\left(u_{1}(t)\right)(z(0))\right]_{x}(0, t)-\left(u_{2}(t)\right)(z(0))_{x}\left(z_{0}, t\right)\right\}+u(t) \nabla g(t, 0)\right\} d t,
\end{aligned}
$$

for all $u \in U$.

Note that $V$ is compact and so is bounded, and that $\nabla g(\cdot, 0)$ is continuous and hence bounded on $\bar{I}$. Next we know that $\hat{\phi}(0) \in L_{2}(Q)$ and $z(0) \in X \subset W_{2}^{1,1}(Q)$. Hence it follows that $J$ is bounded from below on $u$. Then, by a similar argument as that given for Theorem 6.1 of [4], we have:

THEOREM 2.2. Problem (P) has a solution.

REMARK 2.4. From this modified argument, it is easy to observe that:

(i) $U$ is sequentially compact in the weak * topology;

(ii) if a minimizing sequence of controls $\left\{u^{k}\right\} \subset u$ converges to $u^{*} \in U$ in the weak* topology, then $u^{*}$ is an optimal control.

\section{An algorithm}

To devise a computational algorithm for solving Problem ( $P$ ), we need to introduce an auxiliary optimal control problem which can be solved completely.

To begin, let $\hat{h}(x, t)$ and $\hat{g}(t)$ be real-valued continuous functions defined on $Q$ and $I$ respectively. Let $\hat{\phi}(u)$ denote the generalized solution of the system (3) corresponding to the admissible control $u \in U$. Then our auxiliary optimal control problem, referred to as Problem (PI), is :

subject to the system (1), find a control $u \in U$ that minimizes the cost functional

$$
J_{1}(u)=\iint_{Q} \hat{h}(x, t) \hat{\phi}(u)(x, t) d x d t+\int_{I} \hat{g}(t) u(t) d t .
$$

The corresponding adjoint system becomes 


$$
\begin{aligned}
F^{*}(z)(x, t)=\hat{h}(x, t), & (x, t) \in Q, \\
z(x, T)=z_{t}(x, T)=0, & x \in \Omega, \\
z(x, t)=0, & (x, t) \in \Sigma .
\end{aligned}
$$

Then, for any $u \equiv\left(u_{1}, u_{2}\right)$ and $w \equiv\left(w_{1}, w_{2}\right)$ in $u$, it is easily seen that

(II) $J_{1}(u)-J_{1}(w)=\int_{I}\left\{c\left\{\left(u_{1}(t)-w_{1}(t)\right) z_{x}(0, t)-\left(u_{2}(t)-w_{2}(t)\right) z_{x}\left(z_{0}, t\right)\right\}\right.$ $+(u(t)-w(t)) \hat{g}(t)] d t$

where $z$ is the generalized solution of the adjoint system (10).

It follows readily from Corollary 2.1 that:

THEOREM 3.1. Consider Problem (PI) and let $u^{*} \equiv\left(u_{1}^{*}, u_{2}^{*}\right) \in u$. Then the necessary and sufficient condition for $u^{*}$ to be an optimal control is that the inequality

$$
\begin{aligned}
c u_{1}^{*}(t) z_{x}(0, t)-c u_{2}^{*}(t) z_{x}\left(z_{0}, t\right) & +u^{*}(t) \hat{g}(t) \\
\leq & \leq v_{1} z_{x}(0, t)-c v_{2} z_{x}\left(z_{0}, t\right)+v \hat{g}(t),
\end{aligned}
$$

holds for almost all $t \in I$ and for all $v \equiv\left(v_{1}, v_{2}\right) \in V$.

Next, we have:

THEOREM 3.2. Consider Problem (PI). Then there exists an admissible control $u^{*} \equiv\left(u_{1}^{*}, u_{2}^{*}\right) \in u$ such that

$$
\begin{aligned}
c u_{1}^{*}(t) z_{x}(0, t)-c u_{2}^{*}(t) z_{x}\left(z_{0}, t\right)+u^{*}(t) \hat{g}(t) \\
\quad=\inf _{v \equiv\left(v_{1}, v_{2}\right) \in V}\left\{c v_{1} z_{x}(0, t)-c v_{2} z_{x}\left(z_{0}, t\right)+v \hat{g}(t)\right\} .
\end{aligned}
$$

Moreover, this control $u^{*}$ is an optimal control.

The proof of this existence theorem is similar to that given for Theorem 5.2 of [4].

We are now in a position to devise a computational method for solving Problem $(P)$. For this we need certain additional conditions on the functions $h$ and $g$. We assume that:

(i) $\nabla^{2} h(\cdot, \cdot, \phi(u)(\cdot, \cdot))$ is essentially bounded on $Q$, for 
all $u \in U$;

(ii) $\nabla^{2} g(\cdot, u(\cdot))$ is essentially bounded on $I$, for all $u \in u$.

To simplify our presentation, we will introduce some notation. Define $E$ to be the product space

$$
E \equiv L_{2}\left(Q, R^{2}\right) \times L_{2}\left(I, R^{2}\right)
$$

with inner product of two elements

$$
z_{1} \equiv\left(H_{1}, G_{1}\right) \text { and } z_{2} \equiv\left(H_{2}, G_{2}\right)
$$

in $E$ given by

$$
\left\langle z_{1}, Z_{2}\right\rangle \equiv \iint_{Q} H_{1} H_{2} d x d t+\int_{I} G_{1} G_{2} d t
$$

For any $u \in u, z_{u}$ denotes the element

$$
z_{u} \equiv(\hat{\phi}(u)(x, t), u(t))
$$

in $E$, and for any $u, w \in U, \nabla J(u)$ and $\nabla^{2} J(u) z_{w}$ denote the following elements in $E$ :

$$
\begin{aligned}
\nabla J(u) & \equiv(\nabla h(x, t, \phi(u)(x, t)), \nabla g(t, u(t))) \\
\nabla^{2} J(u) z_{w} & \equiv\left(\nabla^{2} h(x, t, \phi(u)(x, t)) \hat{\phi}(w)(x, t), \nabla^{2} g(t, u(t)) w(t)\right) .
\end{aligned}
$$

Consider Problem (P). Then a minimizing sequence of admissible controls $\left\{u^{k}\right\}$ can be generated by the following iterative procedure:

Step 1. Choose an initial control $u^{1} \in U$ and set $k=1$.

Step 2. If $u^{1}, \ldots, u^{k} \in u$ have been chosen, choose $z^{k} \in u$ by the requi rement that

$$
\begin{aligned}
& \text { (18) }\left\langle\nabla J\left(u^{k}\right), z_{\tilde{u}^{k}}\right\rangle \\
& =\min _{u \in U}\left\langle\nabla J\left(u^{k}\right), z_{u}\right\rangle \\
& =\min _{u \in u}\left\{\iint_{Q} \hat{\phi}(u)(x, t) \nabla h\left(x, t, \phi\left(u^{k}\right)(x, t)\right) d x d t+\int_{I} u(t) \nabla g\left(t, u^{k}(t)\right) d t\right\} .
\end{aligned}
$$


Step 3. Let $u^{k+1}=u^{k}+\alpha^{k}\left(\tilde{u}^{k}-u^{k}\right)$ where $\alpha^{k}$ is determined by the requirement that

$$
J\left(u^{k}+\alpha^{k}\left(\tilde{u}^{k}-u^{k}\right)\right)=\inf _{0 \leq \alpha \leq 1} J\left(u^{k}+\alpha\left(\tilde{u}^{k}-u^{k}\right)\right) .
$$

Step 4. Go to Step 2 with $k:=k+1$.

REMARK 3.1. The existence and the construction of $\tilde{u}^{k}$, in step 2 , is guaranteed by Theorem 3.2.

THEOREM 3.3. Consider Problem (P) and let $u^{*}$ be an optimal control and $\left\{u^{k}\right\}$ a sequence of admissible controls generated by the algorithm. Then $\left\{u^{k}\right\}$ is a minimizing sequence; that is,

$$
\lim _{k \rightarrow \infty} J\left(u^{k}\right)=J\left(u^{*}\right)
$$

The proof is similar to that given for Theorem 3.1 of [3].

In closing the paper, we will present some results concerning certain convergence properties of the algorithm. The following result follows readily from Theorem 3.3 and Remark 2.4 .

THEOREM 3.4. The sequence $\left\{u^{k}\right\}$, generated by the algorithm, has a subsequence converging to an optimal control in the weak* topology of $L_{\infty}\left(I, R^{2}\right)$. Moreover, if $u^{*} \in U$ is an accumulation point of the sequence $\left\{u^{k}\right\}$ with respect to the weak* topology, then it is an optimal control.

Finally, we have

THEOREM 3.5. Suppose that, for almost all $(x, t) \in Q, t \in I$, the function $h(x, t, \cdot)$ and $g(t, \cdot)$ are strictly convex in $\phi$ and $u$ respectively. Then the sequence $\left\{u^{k}\right\}$, generated by the algorithm, converges to the optimal control in the weak* topology.

The proof is similar to that given for Theorem 7.3 of [4]. 


\section{References}

[1] N.U. Ahmed and K.L. Teo, Optimal control of distributed parameter systems (North Holland, Amsterdam; Elsevier, New York; 1981).

[2] N.U. Ahmed, "Optimal control of generating policies in a power system governed by a second order hyperbolic partial differential equation", SIAM J. Control. Optim. 15 (1977), 1016-1033.

[3] Earl R. Barnes, "An extension of Gilbert's algorithm for computing optimal controls", J. Optim. Theory Appl. 7 (1971), 420-443.

[4] K.G. Choo, K.L. Teo and Z.S. Wu, "On an optimal control problem involving first order hyperbolic systems with boundary controls", Numer. Funct. Anal. Optim. 4 (1981/82), 171-190.

\footnotetext{
Department of Pure Mathematics,

University of Sydney,

Sydney,

New South Wales 2006,

Australia;

School of Mathematics,

University of New South Wales,

PO Box 1,

Kensington,

New South Wales 2003,

Australia;

Department of Mathematics,

Zhongshan University,

Guanchou,

China.
} 